\title{
The Correlation Between Severity of Postoperative Hypocalcemia and Perioperative Mortality in Chromosome 22q11.2 Microdeletion (22q11DS) Patient After Cardiac-Correction Surgery: A Retrospective Analysis
}

\author{
Chi Yang, MD, PhD,${ }^{1,2}$ Junwen Ge, MD,${ }^{1}$ Rufang Zhang, MD,${ }^{1}$ Chang Chen, $\mathrm{MD}, \mathrm{PhD},{ }^{3}$ Long Yi, MD, \\ $\mathrm{PhD},{ }^{4} \mathrm{Li}$ Shen, $\mathrm{MD}, \mathrm{PhD}^{1}$ \\ ${ }^{1}$ Department of Cardiothoracic Surgery, Shanghai Children's Hospital, Shanghai Jiao Tong University, Shanghai, China; ${ }^{2}$ Clinic and \\ Research Center of Tuberculosis, Shanghai Key Lab of Tuberculosis, Shanghai Pulmonary Hospital, Tongji University School of \\ Medicine, Shanghai, China; ${ }^{3}$ Department of Thoracic Surgery, Shanghai Pulmonary Hospital, Tongji University, Shanghai, China; \\ ${ }^{4}$ Department of Pathology, Nanjing University Medical School, Nanjing, China
}

\section{ABSTRACT}

Objectives: The aim of our study was to elucidate the association between severity of postoperative hypocalcemia and the prognosis of the patients with 22q11DS.

Methods: Data retrospectively were collected from 23 children with 22q11DS who underwent cardiac correction surgery. Area under the receiver operating characteristic curve (AUC) and diagnostic odds ratio were calculated to determine the tendency of perioperative mortality rate, according to the minimum levels of serum calcium and the duration of hypocalcemia. A novel risk assessment system for perioperative mortality was established according to these valid parameters.

Results: The death group had lower minimum levels of serum calcium and longer duration of hypocalcemia. The AUC of minimum levels of serum calcium was $0.912(95 \%$ CI: $0.753-1 ; P=.003)$ and qualified its high accuracy for perioperative mortality. The AUC of duration of hypocalcemia was 0.804 (95\% CI: $0.561-1 ; P=.03)$ and qualified its moderate accuracy. The tendency analyses also indicated the correlation between these two parameters and perioperative mortality. Based on the cut-off values from ROC analysis, a novel risk assessment system for perioperative mortality was established according to these two parameters. The patients with the lowest serum calcium level $<0.885 \mathrm{mmol} / \mathrm{L}$ or duration of the hypocalcemia $>90.33$ hours would be sorted into a high-risk group; others were divided into a low-risk group. The diagnostic odds ratio for this assessment system was 143(95\% CI: 5.13-3982.52). No significant difference was found with regard to patient age, weight, preoperative serum total calcium, cardiopulmonary bypass (CPB) time, and aortic cross-clamp time between the high- and low-risk groups.

Conclusions: The minimum levels of serum calcium and duration of hypocalcemia were valid predictors for preoperative mortality of 22q11DS patients.

Received March 11, 2020; accepted May 25, 2020.

Correspondence: Long Yi and Li Shen (e-mails: yilong@nju.edu.cn, drshen_ shjtu@163.com).

\section{INTRODUCTION}

Chromosome 22q11.2 deletion syndrome (22q11DS), also known as DiGeorge syndrome (OMIM \#188400), is one of the most common chromosomal disorders. The prevalence of 22q11DS is approximately 1:4000-1:6000 in child patients. Multiple complications have been considered to be associated with 22q11.2 deletion syndrome, including immunodeficiency, conotruncal cardiac abnormality, hypocalcemia, dysmorphic facies, speech delay, and velopharyngeal incompetence [Goodship 1998; Kobrynski 2007].

As a common neonate complication of 22q11DS, hypocalcemia may be caused by diminished parathyroid hormone reserve, with an estimated $60 \%$ occurrence in patients with 22q11DS [Ryan 1997]. We previously showed that a significant lower level of serum calcium was found in patients with 22q11DS after cardiac correction surgery, and we also observed decreased postoperative calcium level could result in increased postoperative complications and preoperative mortality [Shen 2011].

In this study, we try to define the relationship between the preoperative mortality and two characteristic features about the severity of postoperative hypocalcemia: minimum serum calcium level and its duration. We carried out a ROC analysis, aiming to elucidate the association between these features and the prognosis of the patients. If these features were classified as relevant factors with prognosis, a classification system criterion base on them would be established.

\section{MATERIALS AND METHODS}

From 2003 to 2012, 23 children underwent cardiac correction surgery in our unit and were confirmed with 22q11DS. To determine the association between serum calcium level and prognosis in 22q11DS patients, we retrospectively collected medical record data from these patients. Our study was performed with approval from the Nanjing University, in accordance with the ethical standards laid down in the 1964 Declaration of Helsinki and its later amendments. 

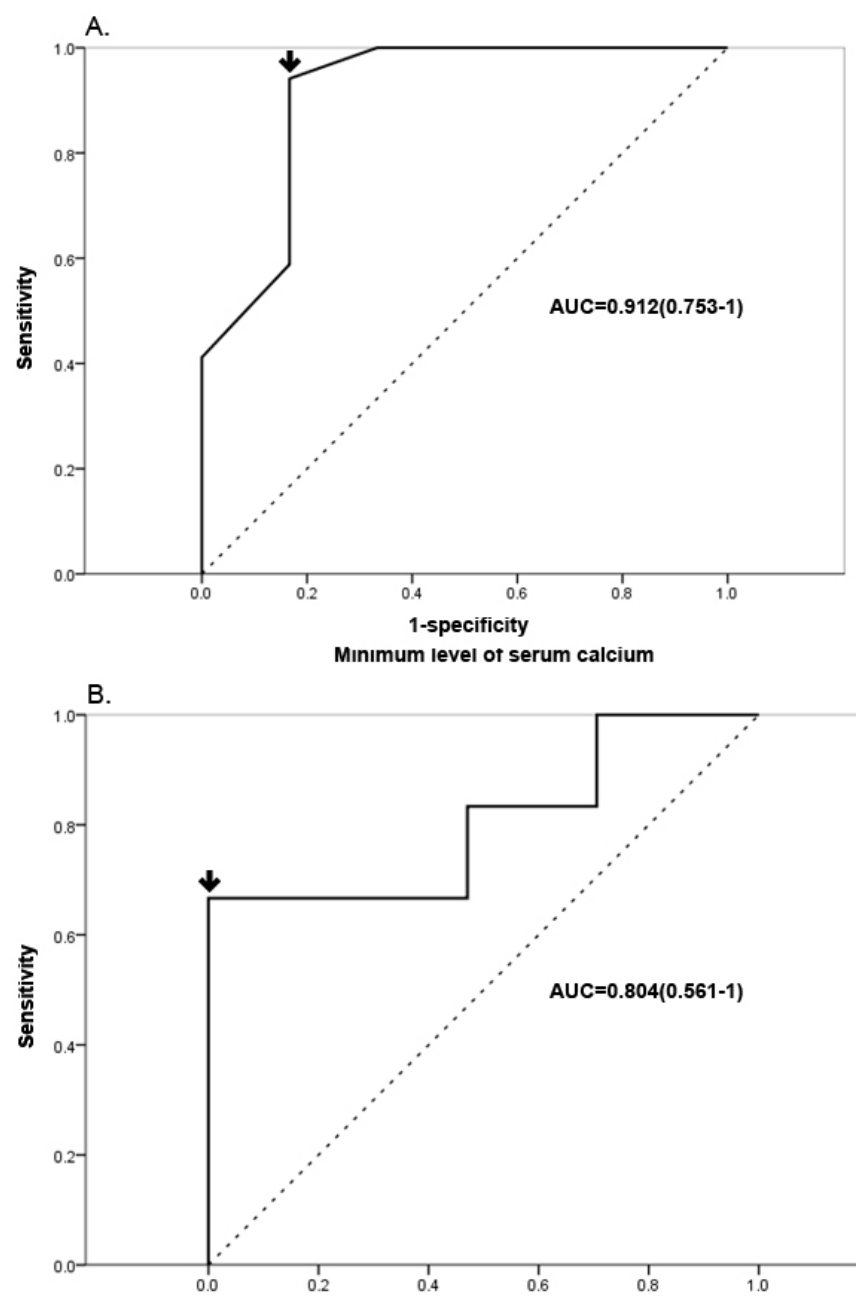

Figure 1. Receiver operating characteristic (ROC) curves for minimum levels of serum calcium (A) and duration of hypocalcemia (B). The dotted diagonal line indicates no discrimination. The optimal cut-off point in $\mathrm{ROC}$ is marked with arrow.

Clinical diagnosis of the 22q11DS was confirmed by a combined use of competitive fluorescent multiplex strip assay (CFMSA) and haplotypes analysis or fluorescence in situ hybridization 22. The protocol of CFMSA was described in a previous report [Yang 2009].

Without a standard protocol for monitoring and treating of hypocalcemia after cardiac surgery, we followed an empirical procedure about calcium management. The measurement of the serum calcium level was performed at preoperation, every three hours on the first day of postoperation, and every 24 hours after the first day of postoperation. When the patient was presented with clinical manifestations of hypocalcemia, an extemporaneous test immediately was administered to confirm the condition. After confirmation with the hypocalcemia (serum calcium level $<1.12 \mathrm{mmol} / \mathrm{L}$ ) [de Andrade 2010], the patient then was applied with $10 \%$
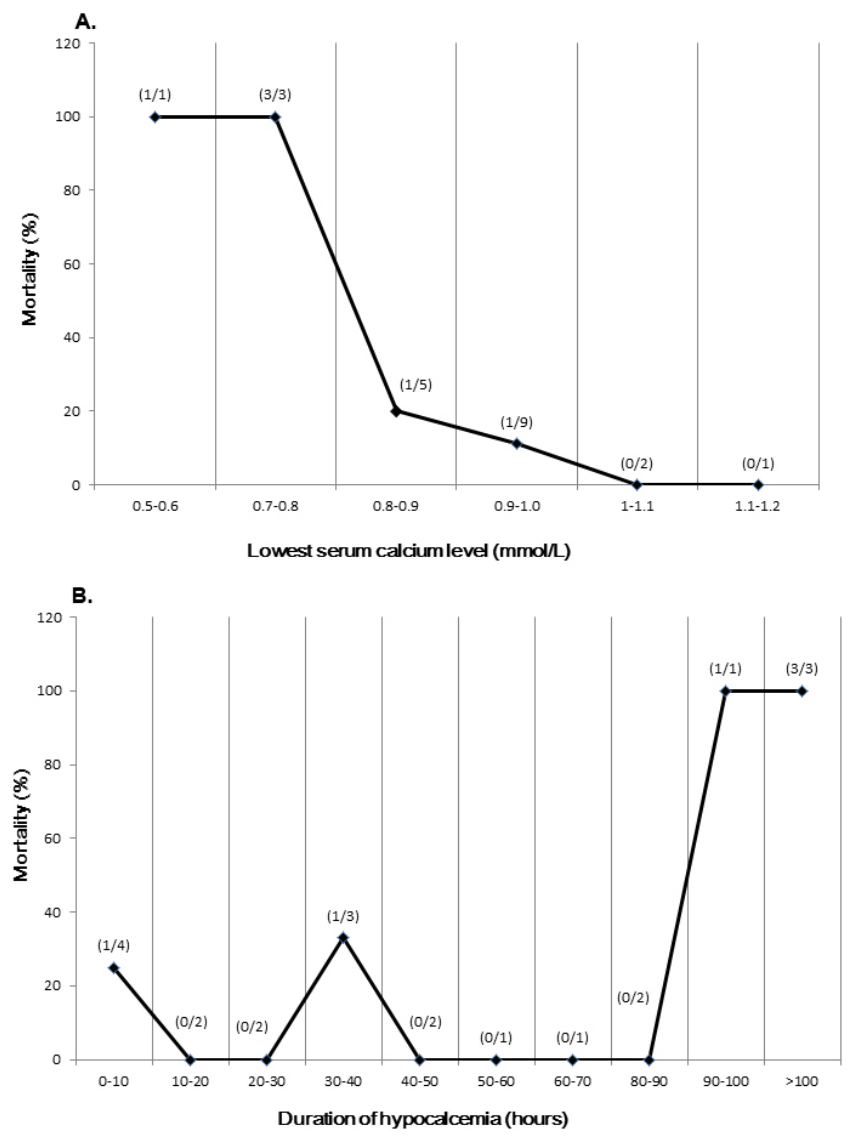

Figure 2. Distribution of the mortality rates in each subgroup according to minimum levels of serum calcium (A) and duration of hypocalcemia (B). The death number and total number for each subgroup were shown in brackets.

calcium gluconate $(0.5 \mathrm{mg} / \mathrm{kg})$ at a time interval of three hours until the recovery of serum calcium level. The duration of serum calcium was defined as the summary time of serum calcium level below normal.

First, the minimum levels of serum calcium and the duration of hypocalcemia are compared between the death group and survival group to assess their potential to predict the probability of death. Then, the ROC curves are calculated and areas under the ROC curve (AUC) of these variables are evaluated to assess their prediction power for preoperative mortality rate. Generally, larger AUC means more reliability and better discrimination [Oh 1993]. Greater than 0.9 means high accuracy, $0.7-0.9$ indicates moderate accuracy, $0.5-0.7$, low accuracy, and 0.5 a chance result [Fischer 2003]. The variables with AUC greater than 0.7 would be accepted as valuable predictors for preoperative mortality rate and their corresponding optimal cut-off values, which maximize the sum of sensitivity and specificity, are calculated. With these cut-off values, new classification methods are established and corresponding sensitivity, specificity, positive predictive value (PPV), negative predictive value (NPV) and diagnostic odds ratio (DOR) 
Table 1. Demographic and clinical characteristics of patients with participants

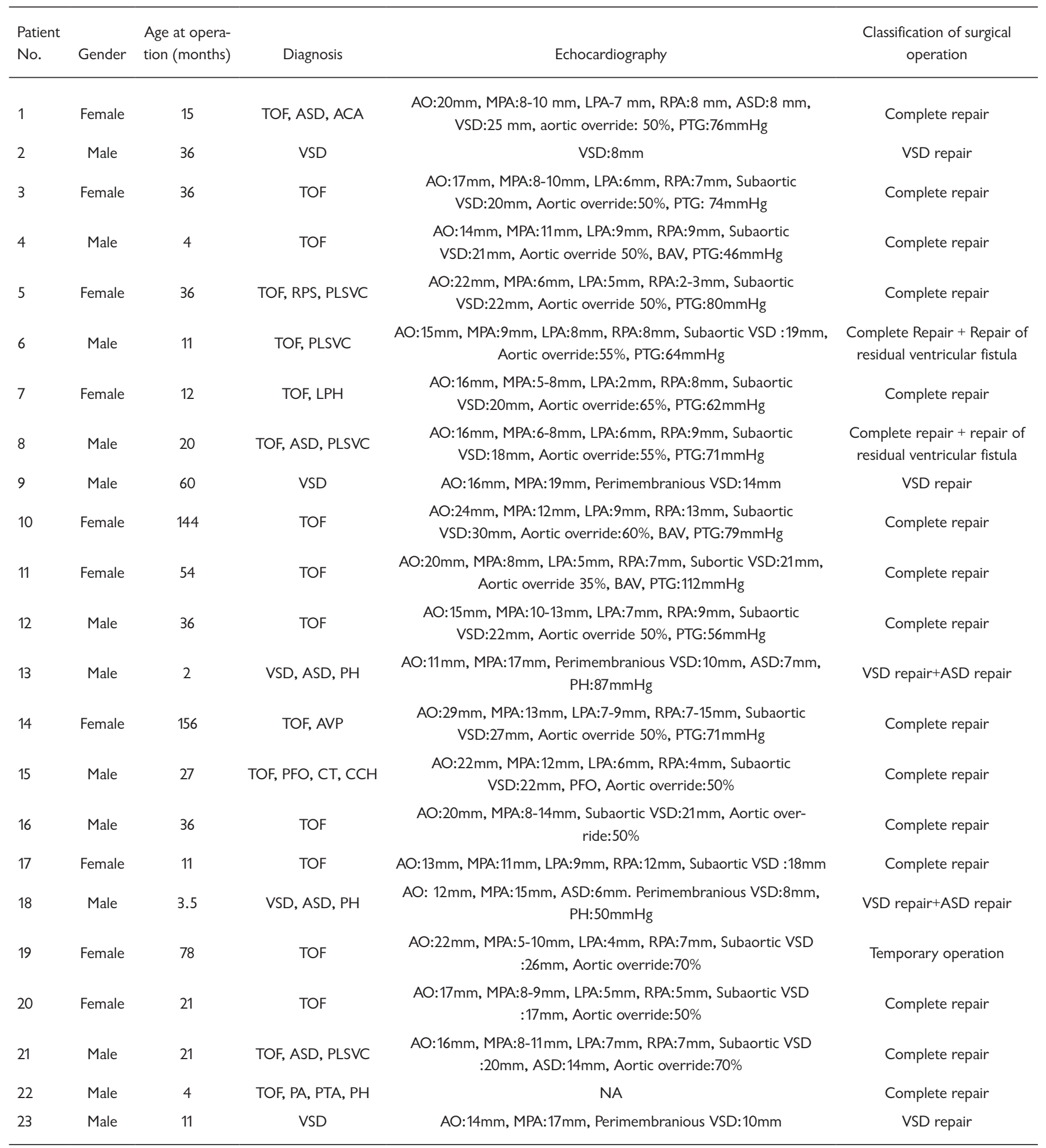

TOF = Tetralogy of Fallot; VSD = ventricular septal defect; ASD = atrial septal defect; ACA = anomalous coronary artery crossing; PLSVC = persistent left superior venacava; $\mathrm{LPH}=$ left pulmonary hypoplasia; $\mathrm{BAV}=$ bicuspid aortic valve; $\mathrm{AVP}=$ aortic valve prolapse; $\mathrm{CT}=\mathrm{Cor}$ triatriatum; $\mathrm{CCH}=\mathrm{criss}-\mathrm{cross}$ heart; $\mathrm{PA}$ = pulmonary artery; $\mathrm{PH}=$ pulmonary hypertension; $\mathrm{MPA}=$ main pulmonary artery; LPA = left pulmonary artery; RPA = right pulmonary artery; PTG = pulmonary transvalvular gradient; $\mathrm{PFO}=$ patent foramen ovale; PTA = persistent truncus arteriosus; NA = not available 
Table 2. Comparison of physiological variables between death and survivor

\begin{tabular}{lcc}
\hline Variables & Death group $(95 \% \mathrm{Cl}) \mathrm{N}=6$ & Survival group $(95 \% \mathrm{Cl}) \mathrm{N}=17$ \\
\hline Minimum levels of serum calcium $(\mathrm{mmol} / \mathrm{L})$ & $0.7700(0.6450-0.8740)$ & $0.9367(95 \% \mathrm{Cl}: 0.9046-0.9723)$ \\
Duration of hypocalcemia (hour) & $174.37(95 \% \mathrm{Cl}: 40.91-324.18)$ & $42.98(95 \% \mathrm{Cl}: 28.26-56.97)$
\end{tabular}

Table 3. Comparison of the Classification methods for preoperative mortality rate

\begin{tabular}{|c|c|c|c|c|c|}
\hline Minimum level of serum calcium ( $<0.855 \mathrm{mmol} / \mathrm{L})$ & $83 \%(5 / 6)$ & $94.2 \%(16 / 17)$ & $83 \%(5 / 6)$ & $94.2 \%(16 / 17)$ & $80(4.20-1525.59)$ \\
\hline Combined method ( $<0.855 \mathrm{mmol} / \mathrm{L}$ or $>90.33$ hours $)$ & $100 \%(6 / 6)$ & $94.2 \% 16 / 17$ & $85.6 \%(6 / 7)$ & $100 \%(15 / 15)$ & $143(5.13-3982.52)$ \\
\hline
\end{tabular}

$\mathrm{PPV}=$ positive predictive value; $\mathrm{NPV}=$ negative predictive value; $\mathrm{DOR}=$ diagnostic odds ratio; $\mathrm{Cl}=$ confidence interval

are calculated. Moreover, the baseline data and operative data from subgroups are compared with the Fisher exact test for categorical variables and one-way ANOVA test for continuous variables. $P<.05$ is considered statistically significant. All the statistical analyses are performed using MedCalc software (v9.0.1.1; MedCalc Software, Mariakerke, Belgium).

\section{RESULTS}

Twenty-three patients with del22q11.2 were included in this study - 10 girls and 13 boys. The median age was 21 (range, 2-156) months. Eighteen patients were diagnosed as Tetralogy of Fallot and most of them (17/18) underwent complete repair. The remaining five patients were diagnosed with ventricular septal defect; all underwent ventricular septal defect repair. Preoperative hypocalcemia was identified in $13.0 \%(3 / 23)$ of patients. The demographic and clinical characteristics of all participants were shown in Table 1 .

To determine whether the minimum levels of serum calcium and the duration of hypocalcemia have the potential to predict the probability of death, a comparison study was performed between the survival group and death group. The minimum serum calcium for the death group statistically was lower than the survival group, and the mean duration of hypocalcemia of the death group also significantly exceeded the survival group (Table 2).

The receiver operating characteristic curves of minimum levels of serum calcium and duration of hypocalcemia about preoperative mortality were shown in Figure 1. Both physiological variables had good discrimination power with AUC $>0.7$. However, minimum levels of serum calcium (AUC $=0.912 ; 95 \%$ CI: $0.753-1 ; P=.003$ ) had better results than duration of hypocalcemia (AUC $=0.804 ; 95 \%$ CI: 0.561-1; $P=.03$ ) in predicting preoperative mortality. The receiver operating characteristic curve also provided the optimal cutoff values $(0.855 \mathrm{mmol} / \mathrm{L}$ for minimum level of serum calcium and 90.33 hours for duration of hypocalcemia), and the corresponding sensitivity, specificity, PPV, NPV, and DOR were shown in Table 3. Minimum level of serum calcium also was more accurate in DOR.

The distribution of preoperative mortality rate according to minimum level of serum calcium and duration of hypocalcemia was analyzed and shown in Figure 2. The interval between adjacent subgroups was $0.1 \mathrm{mmol} / \mathrm{L}$ or 10 hours (Figure 2). In general, preoperative mortality rate tended to increase as minimum level of serum calcium decreased and decrease as duration of hypocalcemia decreased.

For minimum levels of serum calcium, the preoperative mortality rates stayed at high levels (100\%) for patients with serum calcium below 0.8 . However, between 0.8 and 0.9 , the preoperative mortality rate decreased sharply to $20 \%$ and decreased slowly to $0 \%$ when the serum calcium was extended to 1.0 .

For duration of hypocalcemia, when duration was below 90 hours, the preoperative mortality rates generally maintained extremely low levels except the subgroup $(0-10)$ and (30-40). But it showed a steeper increase to $100 \%$, when duration ranged between 90 to 100 .

Since minimum levels of serum calcium and duration of hypocalcemia both were good predictors for preoperative mortality, combining them may further improve the discrimination power. Therefore, we established a combined method to classify patients into two groups, according to both physiological variables as follows: 1) low-risk group (lowest serum calcium level $>0.885 \mathrm{mmol} / \mathrm{L}$ and duration of the hypocalcemia $<90.33$ hours; 2) high-risk group (lowest serum calcium level $<0.885 \mathrm{mmol} / \mathrm{L}$ or duration of the hypocalcemia $>$ 90.33 hours. The corresponding sensitivity, specificity, PPV, NPV, and diagnostic odds ratio for this combined method were shown in Table 4 . This combined method had better performances at diagnostic odds ratio than either physiological variables.

To assess the risk factor for the patients into the high-risk group of the combined method, the baseline data and operative data were compared by using Chi-squared test. No significant 
Table 4. Comparison of demographic and biochemical characteristics between study groups

\begin{tabular}{|c|c|c|c|}
\hline Variables & Low-risk $N=16$ & High-risk $N=7$ & $P$ \\
\hline Age (month) & $39.2(13.5-54)$ & $32.9(11-21)$ & .786 \\
\hline Weight (kg) & $11.6(7.25-14.75)$ & $11.5(8.25-13.75)$ & .986 \\
\hline $\begin{array}{l}\text { Preoperative serum to- } \\
\text { tal calcium (mmol/L) }\end{array}$ & $2.39(2.30-2.52)$ & $2.33(2.03-2.58)$ & .713 \\
\hline CPB time (min) & $69.5(51-77)$ & $92.67(72-95)$ & .239 \\
\hline $\begin{array}{l}\text { Aortic cross-clamp } \\
\text { time (min) }\end{array}$ & $45.25(32.75-50)$ & $60.67(46-73)$ & .349 \\
\hline
\end{tabular}

The data here was expressed as median (interquartile range); CPB, cardiopulmonary bypass. $\mathrm{NA}=$ not available

difference was found with regard to patient age, weight, preoperative serum total calcium, cardiopulmonary bypass (CPB) time, and aortic cross-clamp time between the groups.

\section{DISCUSSION}

The hypocalcemia of $22 \mathrm{q} 11.2$ DS patients manifests during the neonatal and adolescent stages, decreases in severity after the neonatal period, enters latency later in childhood, and may become apparent again during adolescence [Ryan 1997; Taylor 2003; Greig 1996; Adachi 1998]. However, the physical and emotional stress experienced before and during surgery may increase calcium demand and cause hypocalcemia [Cuneo 1996; Schaan 2006]. Previous study already has shown postoperative hypocalcemia occurs very frequently (86.4-94.1\%) during postoperative course among $22 \mathrm{q} 11.2$ DS patients, and a higher preoperative mortality in $22 \mathrm{q} 11.2$ DS patients with hypocalcemia compared with the $22 \mathrm{q} 11.2$ DS patients was revealed [Shen 2011; Cuturilo 2017]. Furthermore, in this article, we suggested that severity of hypocalcemia also was related to preoperative mortality, and lower serum calcium level and longer duration of hypocalcemia indicated higher preoperative mortality. Our ROC analysis qualified these two parameters as more efficacious predictors than merely considering occurrence of hypocalcemia.

In view of the possible adverse consequences of hypocalcemia following cardiac surgery, there are strong reasons for restoring normal levels as quickly as possible. Currently, calcium supplementation is conventional treatment for $22 \mathrm{q} 11 \mathrm{DS}$ patients with hypocalcemia [Choi 2005]. However, the efficacy of the calcium supplementation also varies between different individuals from $22 \mathrm{q} 11 \mathrm{DS}$ patients. Our preliminary data showed that some patients from the high-risk group could not be improved even with a high dosage of calcium infusion. In those patients, their hypocalcemia symptoms persisted even with a high dosage of $16.1 \mathrm{mg} / \mathrm{kg} / \mathrm{h}$ calcium supplementation. This uncorrectable hypocalcemia suggests a different etiology and further studies are necessary to unveil the mechanism of the symptoms for these patients.
Previous studies [Robertie 1991; Robertie 1992] have shown that appropriate responses of calcium-magnesiumparathyroid hormone-calcitriol axis during CPB surgery was critical for the ionized calcium level. In patients without 22q11DS, ionized calcium level usually was decreased after the initiation of $\mathrm{CPB}$, and persistent hypocalcemia eventually could result in maximal response of the PTH system, and a normal ionized calcium level could be achieved due to the PTH system. However, a spontaneous recovery of the ionized calcium level could not be achieved in 22q11DS patients, due to the deficiency of the PTH [Cuneo 1996; Kapadia 2008]. Therefore, appropriate regulation of the PTH system, during the postoperative phase, could be considered a solution to hypocalcemia symptoms. However, further investigation should be performed to test the change of the level PTH at pre- and postoperation.

The serious hypocalcemia was common following elective cardiac surgery, occurring in $30.4 \%$ of patients, but its reason was not obvious. To determine the possible causes for the high-risk group, the association between groups and variables such as age, weight, CPB time, and aortic cross-clamp time independently were analyzed. However, no significant association was found with these variables among the patients in the different groups. Further analysis of other risk factors should be performed.

The main disadvantage of this study is the small number of patients. Additionally, the loss of some baseline variables, such as immunodeficiency status, restricted the statistical power of our study. Therefore, a large number of patients might be recruited to confirm the results.

\section{CONCLUSION}

This study identified serum calcium and the duration of hypocalcemia as valuable predictors for preoperative mortality and established a novel risk assessment system for preoperative mortality, according to these two parameters. Concern with the adverse consequences of the high-risk group and the resistance to traditional calcium supplement indicates that the development for new therapy urgently is needed.

\section{REFERENCES}

Adachi M, Tachibana K, Masuno M, et al. 1998. Clinical characteristics of children with hypoparathyroidism due to $22 \mathrm{q} 11.2$ microdeletion. European Journal of Pediatrics 157:34-38.

Choi JH, Shin YL, Kim GH, et al. 2005. Endocrine manifestations of chromosome 22q11.2 microdeletion syndrome. Hormone Research 63:294-299.

Cuneo BF, Langman CB, Ilbawi MN, et al. 1996. Driscoll DA. Latent hypoparathyroidism in children with conotruncal cardiac defects. Circulation 93:1702-1708.

Cuturilo G, Drakulic D, Jovanovic I, et al. 2017. The Impact of 22q11.2 Microdeletion on Cardiac Surgery Postoperative Outcome. Pediatric Cardiology. 38: 1680-1685. 
de Andrade Sousa A, Salles JM, Soares JM, et al. 2010. Course of ionized calcium after thyroidectomy. World Journal of Surgery 34:987-992.

Fischer JE, Bachmann LM, Jaeschke R. 2003. A readers' guide to the interpretation of diagnostic test properties: clinical example of sepsis. Intensive Care Medicine 29:1043-1051.

Goodship J, Cross I, LiLing J, et al. 1998. A population study of chromosome 22q11 deletions in infancy. Archives of Disease in Childhood 79:348-351.

Greig F, Paul E, DiMartino-Nardi J, et al. 1996. Transient congenital hypoparathyroidism: resolution and recurrence in chromosome 22q11 deletion. The Journal of Pediatrics 128:563-567.

Kapadia CR, Kim YE, McDonald-McGinn DM, et al. 2008. Parathyroid hormone reserve in 22q11.2 deletion syndrome. Genetics in Medicine: Official Journal of the American College of Medical Genetics 10:224-228.

Kobrynski LJ, Sullivan KE. 2007. Velocardiofacial syndrome, DiGeorge syndrome: the chromosome $22 \mathrm{q} 11.2$ deletion syndromes. Lancet 370:1443-1452.

Oh TE, Hutchinson R, Short S, et al. 1993. Verification of the Acute Physiology and Chronic Health Evaluation scoring system in a Hong Kong intensive care unit. Critical Care Medicine 21:698-705.
Robertie PG, Butterworth JFt, Royster RL, et al. 1991. Normal parathyroid hormone responses to hypocalcemia during cardiopulmonary bypass. Anesthesiology 75:43-48.

Robertie PG, Butterworth JFt, Prielipp RC, et al. 1992. Parathyroid hormone responses to marked hypocalcemia in infants and young children undergoing repair of congenital heart disease. Journal of the American College of Cardiology 20:672-677.

Ryan AK, Goodship JA, Wilson DI, et al. 1997. Spectrum of clinical features associated with interstitial chromosome 22q11 deletions: a European collaborative study. Journal of Medical Genetics 34:798-804.

Schaan BD, Huber J, Leite JC, et al. 2006. Cardiac surgery unmasks latent hypoparathyroidism in a child with the $22 \mathrm{q} 11.2$ deletion syndrome. Journal of Pediatric Endocrinology \& Metabolism: JPEM 19:943-946.

Shen L, Gu H, Wang D, et al. 2011. Influence of chromosome 22q11.2 microdeletion on postoperative calcium level after cardiac-correction surgery. Pediatric Cardiology 32:904-909.

Taylor SC, Morris G, Wilson D, et al. 2003. Hypoparathyroidism and 22q11 deletion syndrome. Archives of Disease in Childhood 88:520-522.

Yang C, Shen L, Xu Z, et al. 2009. A novel competitive fluorescent multiplex STR polymorphism assay for rapid, reliable and single-tube screening of 22q11.2 copy-number aberrations. Electrophoresis 30:465-471. 